\title{
La infiltración con corticoides mejora los síntomas del túnel carpiano
}

Injection with methylprednisolone proximal to the carpal tunnel: randomised double blind trial.

Dammers JWHH, Beering MM, Vermeulen M. BMJ 1999;319:884-6.

\section{Objetivo}

Evaluar el efecto de una infiltración proximal al túnel carpiano (TC) con $40 \mathrm{mg}$ de metilprednisolona en los síntomas del síndrome de túnel carpiano.

\section{Diseño}

Ensayo clínico randomizado doble ciego con un año de seguimiento, seguido por un estudio abierto en los no respondedores del grupo control.

\author{
Lugar \\ Consultorio externo de Neurología en un Hospital General de distrito \\ en Holanda.
}

\section{Pacientes}

Pacientes mayores de 18 años, derivados al Servicio de Neurología, con síntomas de túnel carpiano de más de tres meses de evolución , y diagnóstico confirmado por electromiograma. En los pacientes con síntomas bilaterales se randomizó el lado más severo. Se excluyeron pacientes con tratamiento previo.

\section{Intervención}

Los pacientes fueron randomizados para recibir una infiltración con 10 mg de lignocaina, $010 \mathrm{mg}$ de lignocaina más $40 \mathrm{mg}$ de metilprednisolona. Los no respondedores que habían recibido lignocaina sola recibían $40 \mathrm{mg}$ de metilprednisolona más $10 \mathrm{mg}$ de lignocaina en el estudio abierto. La infiltración se realizaba $4 \mathrm{~cm}$ proximal a la muñeca entre los tendones del flexor común de los dedos y el palmar mayor.

\section{Medición de resultados principales}

Los pacientes fueron clasificados en respondedores y no respondedores. Respondedores eran aquellos que no referían síntomas o con síntomas menores que el paciente consideraba no requerían ningún tratamiento adicional. Esto fue evaluado al mes, 3, 6, 9 y 12 meses después de la infiltración. Si se requería tratamiento adicional se abría el código $y$, si el paciente había recibido lignocaina sola, se le ofrecía infiltración con metilprednisona, si no, se realizaba cirugía de descompresión.

\section{Resultados principales}

Al mes, sólo $6(20 \%)$ de los 30 pacientes del grupo control respondieron vs. $23(77 \%)$ de los 30 pacientes del grupo intervención (diferencia observada: $57 \%$; IC 95\%: $36 \%-77 \%$ ). Al año sólo 2 de los 6 pacientes respondedores del grupo control no necesitaron otro tratamiento, vs. 15 de los 23 del grupo intervención (diferencia: $43 \%$; IC $95 \%: 23 \%-63 \%$ ). En todos los intervalos medidos, el número de pacientes respondedores fue significativamente mayor en el grupo intervención.

En el estudio abierto 24 de 28 (86\%) pacientes del grupo control respondieron al tratamiento con metilprednisona, a pesar que 12 de estos 24 requirieron tratamiento quirúrgico dentro del año

\section{Conclusiones}

Una única infiltración con corticoides proximal al túnel carpiano produjo una mejoría a largo plazo en los síntomas de pacientes con síndrome del túnel carpiano y debe ser considerada antes de la cirugía de descompresión.

\section{COMENTARIO}

El síndrome del TC o neuropatía compresiva del nervio mediano en la muñeca, es la más común de las neuropatias compresivas afectando entre el 0,1 y el $0,5 \%$ de la población en EE.UU 1 . Hay gran cantidad de condiciones asociadas a este síndrome (embarazo, diabetes, hipotiroidismo, enfermedades reumáticas inflamatorias, amiloidosis, etc). Los pacientes se quejan de dolor quemante y parestesias en la mano que aparecen por la noche y mejoran al sacudir la misma. Los síntomas sensitivos pueden estar limitados al clásico territorio del mediano: eminencia tenar, y región palmar de los dedos 1 a 4, o puede afectar regiones más amplias o limitadas de la cara palmar. Ocasionalmente el dolor se puede extender en forma proximal, incluso hasta el hombro, dificultando el diagnóstico.

Desde hace muchos años que la infiltración con corticoides es considerada como altamente efectiva (80\%) en el tratamiento del TC 2-3-4. Lo más novedoso de este artículo es el sitio de la infiltración. Los autores aplican la misma $4 \mathrm{~cm}$ por arriba de la muñeca en lugar de sobre el pliegue distal de la misma, como es clásico ${ }^{2-3-4}$. Aducen que esta localización es menos probable que produzca lesión del nervio y más sencilla de realizar, aunque esto no ha sido demostrado en estudios comparando ambos métodos.

El cálculo del tamaño de la muestra necesario para mostrar diferencias fue de 80 pacientes. Sin embargo el comité de ética detuvo el estudio cuando se habían incluido sólo 60 pacientes. A pesar de esto el estudio tuvo un poder del $99 \%$ para detectar diferencias al mes con un nivel de significancia del $5 \%$, debido a que el porcentaje de respuesta en el grupo control fue menor al calculado ( $20 \%$ vs $50 \%$ ).

Otras dos buenas noticias de este estudio son: una mayor duración del beneficio del tratamiento que en estudios previos ${ }^{3-4}$. Estas variaciones seguramente se deben a diferencias en la selección de los pacientes, y sus enfermedades asociadas que no fueron comentadas en el estudio; $y$, que la media de duración de los síntomas fue de más de dos años, lo cual va en contra de que con síntomas de más de un año de duración es esperable una pobre respuesta como se sostiene clásicamente ${ }^{3-4}$.

Como conclusión, este estudio refuerza el concepto que la infiltración con corticoides debe ser considerada como opción previa a la cirugía en el síndrome del túnel carpiano, aún en pacientes con mucho tiempo de evolución. Se presenta una alternativa en el sitio de infiltración, al clásicamente utilizado.

\section{Dr. Enrique Soriano}

Reumatología. Hospital Italiano de Buenos Aires.

\section{Referencias}

1. Stevens JC, Sun S, Beard CM, et al. Carpal tunnel syndrome in Rochester, Minnesota, 1961-1980. Neurology 1998; 38:134-37.

2. Girlanda P, Dattola R, Venuto C, et al. Local steroid treatment in idiophatic carpal tunnel syndrome: short and long term efficacy. J Neurol 1993;240:187-90

3. Gelberman RH, Aronson D, Weisman M. Carpal tunnel syndrome. J Bone Joint Surg 1980;62:1181-4.

4. Weiss APC, Sachar K, Gendreau M. Conservative management of carpal tunnel syndrome: A reexamination of steroid injection and splinting. J Hand Surg 1994;19:410-15. 